\title{
Design of remote smart home control system based on the Internet of Things
}

\author{
Yajin Che \\ Sichuan Vocational College of information Technolog, postcode : 628000
}

\begin{abstract}
With the continuous innovation of related technology and the rapid development of information industry, the Internet of things, as a new product of information technology, has been applied in many fields, especially in the field of residential home. It is of great significance to promote the quality of people's daily life. In view of this, this paper briefly analyzes the collection and transmission of indoor remote control signal. On the basis of this, the design of remote control software is analyzed and discussed. Finally, the practical application test shows that the remote smart home control system designed by this research can realize the effective transmission of the indoor electrical control of information. It can meet the needs of practical use both in controlling accuracy and the delay.
\end{abstract}

\section{GENERAL INSTRUCTION}

Since the advent of the Internet of things, it has been widely used in all aspects of social life, and has brought great changes to people's daily life. The application of Internet of things in the field of home has realized the innovation of design ideas for smart home control system, which brings great convenience to people's daily life and promotes the quality of life. To achieve remote smart home control by using Internet of things, the main work is to use the Internet to achieve the connection of all kinds of household equipment and promote the formation of a more complete internal network environment. Thus it can achieve the automatic control of the equipment and scientific management.

\subsection{Design Concept of Smart Home Control System}

There is a very close relationship between the construction of smart home control system and the gradual improvement of the Internet of things. The application of smart home control system will greatly promote the convenience of people's daily life and improve the quality of life. The design principle of the smart home control system is to implement the remote control and management for the indoor equipment by using the Internet of things technology and the sensor nodes. In essence, the working principle of this system is to achieve the control of equipment based on the external control information which is obtained from the separation and the compressor delivery. To have a good applicability, it is necessary to ensure that access to the external control signal received by smart home control system has a certain reliability and can be connected with control components.
And then the remote intelligent control and management of the furniture equipment can be achieved.

In addition, there are some specific requirements to be met in the design and application of remote smart home control system, such as obtaining control command sensing its model by using the system, achieving real-time attention, controlling and managing different types of control by using completely different signals, and including a subsystem for signal processing.

\section{TRANSMISSION AND COLLECTION OF INDOOR REMOTE CONTROL SIGNAL}

\subsection{Amplification and Compensation of Remote Signal}

In order to achieve the effective control of the remote smart home, its precision and distance usually have a great influence on the new generation of smart home system. To ensure the stability of the remote control signal, it is necessary to adopt certain measures to realize the amplification of the circuit, and then achieve the effective control of the signal to ensure that there are no serious mistakes. The energy supply of the amplifying circuit control signal mainly includes two categories: the interior and the exterior. It can amplify the remote control signals and greatly improve the control accuracy. This module mainly has the following functions:

1) High sensitivity. It can not only realize the removal of noise, but also enlarge the difference of the remote signal. It is energy-saving. Generally the circuit current is required to be controlled in: 480VA; when in standby operation, the voltage is $2.6 \mathrm{VA}$.

2) In general, the driving voltage will always remain in the range of $2.2 \mathrm{~V} \sim 3.6 \mathrm{~V}$. 
3) The signal value of the voltage change can be obtained in time, and the actual working condition of the electric appliance can be reflected in a timely manner.

4) Low pass filter can be appropriate for the internal signal processing.

If the input control signal is relatively small in the hardware connection, small signal model should be used. FET micro equivalent circuit and small size model of bipolar transistor are basically the same. The field effect pipeline can be regarded as a two port network, and the output interface can be in the range of the drain electrode and the source electrode when the input interface is in the range of the grid electrode and the source electrode. Whatever kind of field effect transistor it is, it can be regarded as zero grid current value. The input interface can be equated to open circuit because of the voltage value between the grid electrode and the source electrode. Thereby it can realize the acquisition of the network and the amplification of the control signal on the bottom, and then convert into a voltage signal.

\subsection{Reception of Remote Signal}

In order to ensure that the signal still can be received timely and accurately when there is certain loss in the process of transmission, in this design, the serial interface and the USB interface are used to collect the remote control signal information data, and transmit it to the corresponding electrical control terminal. The designed devices for receiving signal are as follows:

1) Wireless serial receiver

XL01-232AP1 is a half-duplex wireless transmission module of UART interface, whose general operating band will be in several $\mathrm{Hz}$ bands like $325,760,825$ and so on. This module strictly meets ETSI and FCC15.247/15.249 certificated standards and the relevant requirements of wireless control. This wireless serial receiver uses the serial port to debug the software equipment to carry on the output power, the serial port format, the serial port speed, the frequency of work and so on.

2) Discriminator of Multiple Control Command

In order to be more effective in identifying and dividing the control of the command, WUSB principle needs to be taken. The main connection principle of this part is the network hub and the topology structure. The data information processed by the host machine is sent to each device based on the WUSB host, and at the same time it configures the corresponding address and the loan. Thereby it generates a group relationship between the device and the remote control device. The WUSB host and its corresponding device use the pattern of "Point-to-Point" to achieve directional transmission. WUSB host can achieve the identification of multiple WUSB devices, and at the same time it can be connected to completely different WUSB hosts. When the different types of WUSB devices are in a relatively small space, the most important problem is how to improve the access capability of the equipment and the reasonable utilization of the bandwidth. At this time, we can use the topology to determine the number of devices to be used, and to achieve the expected control effect.
3) Judgment of the Properties of Control Commands

By using wireless serial port command code, it can achieve the distinction of the actual property control. The serial communication mode is: $\mathrm{N}-6-1$, where $\mathrm{N}$ is the initial digit, 6 data bits, 1 stop bit, and no parity. The communication baud rate has always been in the range of 1300 114500bps. When the data packet is sent, it starts with the command "OT02" and ends with the command "OT0d" to reduce the bit error rate as much as possible. The structure of data report command is the initial command, the remote command code, the control signal data, and the control command tail. Table 1 is a set of instructions of remote control device.

Table 1 the specific encoding of control commands

\begin{tabular}{|c|c|c|}
\hline Instruction code & content & meaning \\
\hline $\mathrm{CC}$ & $0 \sim 0 x 11$ & Open \\
\hline DC & 0 0xll & Close \\
\hline DD & 0 0xll & Range of finishing \\
\hline $\mathrm{EC}$ & 0 0xll & $\begin{array}{c}\text { Functional } \\
\text { disconnection }\end{array}$ \\
\hline ED & 0 0xll & Open the alarm \\
\hline $\mathrm{CC}$ & 0 0xll & Shut down the alarm \\
\hline $\mathrm{CD}$ & 0 0x1l & $\begin{array}{l}\text { Disconnect } \\
\text { functions }\end{array}$ \\
\hline
\end{tabular}

\section{DESIGN OF REMOTE CONTROL SOFTWARE}

New smart home control system software can not only indicate whether the signal is safe, but also appropriately process the signal. In order to be more user-friendly and to ensure that the design of the operating interface is more intuitive, the data will be timely and effectively improved and updated. In the software system, the main modules include data storage, data analysis and processing, data display and man-machine interface.

\subsection{Design of Remote signal acquisition}

If the remote control signal is obtained from the sensor network, the control information can be stored and processed. The attributes of the control information include acquisition time, command characteristics, number of the device, and whether it is specific or not. Generally, the more complex control information will have the lower probability of false operation. 


\subsection{Design of the Display of Control Signal's Attribute}

By using this module, it can be more accurate to describe the properties of the control signal, as it uses the voice to play the relevant information. In addition, this software can also put the related data directly into the log, which can be applied to the different time periods of the accurate data records of valve pressure.

\subsection{Design of Accurate Judgment of Control Signal}

This module is the core of the overall software design. The basis of the attribute operation of the control signal is generally related to the wavelet function, and if it is continuous, the moment when " $m$ " order vanishes is of the integer value. When " $t$ " is in the interval $[a, b]$, if " $f(t)$ " wavelet can be transformed as:

$$
\left|W_{2^{j}} f(t)\right| \leq K\left(2^{j}\right)^{a}
$$

The corresponding logarithm shall be taken as:

$$
\log _{2}\left|W_{2^{j}} f(t)\right| \leq \log _{2} K+j \alpha
$$

In this formula, if $\mathrm{K}$ is a constant, then the Lipschitz index between the $[a, b]$ is uniformly a.

If $\mathrm{a}=2 \mathrm{j}$, then the above formula is:

$$
\left|W_{2^{j}} f(t)\right| \leq K\left(2^{j}\right)^{a}
$$

Or:

$$
\log _{2}\left|W_{2^{j}} f(t)\right| \leq \log _{2} K+j \alpha
$$

Table 2 the range of value of the control signal's attribute

\begin{tabular}{|c|c|}
\hline Value range of a & Properties of control signal \\
\hline$m<a<m+1$ & Control signal properties are of a small change, so the $\mathrm{m}+1$ times function cannot micro. \\
\hline$a=1$ & In the critical range of control command, the (t) is slope or the mountain function in a certain width. \\
\hline $0<a<1$ & Under the wrong operation, the function is discontinuous. \\
\hline$a=0$ & The control signal does not change significantly; it is not likely to have control command transmission. \\
\hline$a=-1$ & \\
\hline
\end{tabular}

According to the analysis of the above theory and the control signal corresponding to the attribute value range in table 2, the control command signal changes when the maximum value of the module changes. It can distinguish the noise of the signal, the maximum value caused by misuse, and the control signal that is not affected. Then the partial wavelet coefficients of the noise can be eliminated completely, and the corresponding coefficients of the control signals can be reconstructed effectively.

\section{PRACTICAL APPLICATION TEST}

To test whether the design method proposed by this research is effective, it uses the calculation method to verify the system. The control signal is transmitted and collected according to the different distances from the control signal. In the validation process, completely different attributes and command signals are divided into different groups, in which 3 of them are used to complete the signal control and cooperative transmission. The control signals are sent and collected according to different types. There are two kinds of control command, which are sent and closed. Simulation of command signal is shown in Figure 1 below.

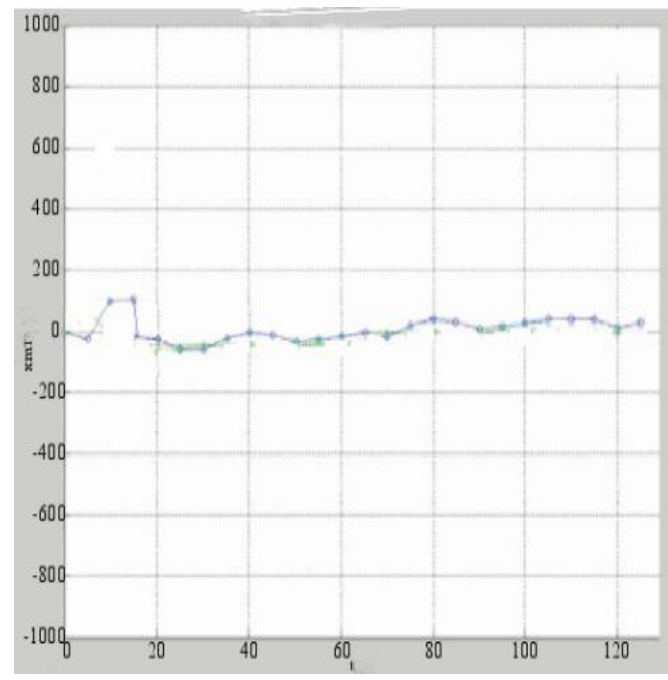

Figure 1 waveform of the command signal

Based on Figure 1, the results show that this system can satisfy the requirements of a certain job on the basis of the transmission and acquisition of multiple command signals. The performance of the system can be satisfied with the general performance requirements, and can be used for receiving and sending multiple models. The performance of the test system is given, which can basically meet the requirements of setting distance, and 
achieve a more satisfactory effect.

Using the remote smart home control system based on the Internet of things, the simulation of the operator bears the starter in the instruction to identify the value of the received instruction information, and then controls and manages various types of accessed electrical equipment according to related instruction. In essence, the role of the simulation of the operator is similar as computer motherboard. It makes specific operation according to the instructions corresponded to the content, so as to achieve the effective control of equipment. The most obvious advantage of remote control is that it is easy to carry and operate. Users only need to integrate the control chip into the phone so that they can use the radio to control the home appliances. And in this process, the remote controller is ensuring that users can transfer the command to the signal receiver promptly and accurately, so as to facilitate the control system to make a more timely and effective response.

\section{CONCLUSION}

With the quickening pace of human life, people need to live and work under increasing pressure. Through the remote smart home control system based on the Internet of things, it can not only alleviates people's work pressure, but also improve their quality of life. However, in view of that the cost of remote smart home control system is relatively high and the operation is also very complex, it is still difficult to achieve a wide range of popularity. Therefore, it is necessary to put forward new design methods to promote the improvement of remote smart home control system, and ensure that the system can play a good role in our daily life.

\section{REFERENCES}

1. Tu Liang, Duan Hongguang. Design of Internet of things smart home system based on 433 wireless transceiver modules [J]. TV Technology, 2012, (6).

2. Meng Xiaoli. The design of intelligent home system based on cloud computing in the Internet of things platform [J]. Science Bulletin, 2016, (6).

3. Xin Hailiang, Zhong Peisi, Zhu Shaoqi et al. Intelligent home control system based on ZigBee [J]. Electronic Technology Applications, 2013, (12).

4. Hu Xiangdong, Han Kaimin, Xu Hongru et al. Safety design and verification of the smart home Internet of things $[\mathrm{J}]$. Journal of Chongqing University of Posts and Telecommunications (NATURAL SCIENCE EDITION), 2014, (2).

5. Deng Yun, Cheng Xiaohui. Design of intelligent home system for the Internet of things $[\mathrm{J}]$. Journal of Guilin University of Technology, 2012, (2).

6. Zhang Guiqing, Lu man, Wang Ming et al. The "spring" of smart home is coming [J]. Computer Science, 2013, (z1).

7. Dong Aimin, Xu Jing. Remote home sensing and control system based on the public cloud platform of Internet of things $[\mathrm{J}]$. Modern Electronic Technology, 2016, (11).

8. Xu Jinqiang. Application of BENQ-M23 GSM/GPRS module in remote intelligent home control[J]. Modern Electronic Technology, 2014, (18): 41-43. 